\title{
THE MYERS-STEENROD THEOREM FOR FINSLER MANIFOLDS OF LOW REGULARITY.
}

\author{
VLADIMIR S. MATVEEV AND MARC TROYANOV
}

\begin{abstract}
We prove a version of Myers-Steenrod's theorem for Finsler manifolds under minimal regularity hypothesis. In particular we show that an isometry between $C^{k, \alpha}$-smooth (or partially smooth) Finsler metrics, with $k+\alpha>0, k \in \mathbb{N} \cup\{0\}$, and $0 \leq \alpha \leq 1$ is necessary a diffeomorphism of class $C^{k+1, \alpha}$. A generalisation of this result to the case of Finsler 1-quasiconformal mapping is given. The proofs are based on the reduction of the Finlserian problems to Riemannian ones with the help of the Binet-Legendre metric.
\end{abstract}

2000 AMS Mathematics Subject Classification: 53b40,53c60,35b65.

Keywords: Finsler metric, isometries, Myers-Steenrod theorem, Binet-Legendre metric.

\section{INTRODUCTION}

The main goal in this paper is to prove a Myers-Steenrod theorem for Finsler manifolds under low regularity assumptions. More precisely, we give an answer to the following question: What is the regularity (smoothness) of a distance preserving bijection $\phi: M_{1} \rightarrow M_{2}$ between two Finsler manifolds $\left(M_{1}, F_{1}\right)$ and $\left(M_{2}, F_{2}\right)$ of low regularity?

This question has been investigated by several authors starting with the seminal 1939 paper by S. Myers and N. Steenrod on Riemannian isometries [22]. We provide a brief historical account at the end of the paper. Our approach in this paper is to reduce the Finsler case to the Riemannian one and to use results on Riemannian isometries proved in [15, 27, 28, 31, 32. Our tool is the Binet-Legendre metric $g_{F}$. It is a Riemannian metric on $M$ canonically associated to $F$ by some averaging procedure, see Section 2.2 below. This metric enjoys some natural geometric properties that makes it a useful tool in Finsler geometry; this is concretely illustrated in [18, 19. We state our main result as the following two Theorems; in these statements, we assume

$$
k \in \mathbb{N} \cup\{0\}, \quad 0 \leq \alpha \leq 1 \text { and } k+\alpha>0 .
$$

Theorem A. Let $\phi: M_{1} \rightarrow M_{2}$ be a distance preserving bijective map between two Finsler manifolds $\left(M_{1}, F_{1}\right)$ and $\left(M_{2}, F_{2}\right)$. Assume that the Binet-Legendre metrics $g_{F_{1}}$ and $g_{F_{2}}$ associated to $F_{1}$ and $F_{2}$ are locally of class $C^{k, \alpha}$. Then $\phi \in$ $C_{l o c}^{k+1, \alpha}\left(M_{1}, M_{2}\right)$ and $\phi^{*}\left(F_{2}\right)=F_{1}$.

Date: May 12, 2016.

We thank the Friedrich-Schiller-Universität Jena, EPFL and the Swiss National Science Foundation for their support. 
Recall that the notation $C^{k, \alpha}$ stands for the class of functions or mappings that are $k$ times continuous differentiable and all of whose partial derivatives of order $k$ are Hölder continuous of order $\alpha$ (or Lipschitz continuous if $\alpha=1$ ).

Theorem B. If a Finsler metric $F$ is of class $C_{l o c}^{k, \alpha}$, then its Binet-Legendre metric is also of class $C_{l o c}^{k, \alpha}$.

Combining these two theorems, we obtain the following:

Corollary C. Any distance preserving bijective map $\phi$ between two manifolds with Hölder continuous Finsler metrics is a diffeomorphism. Moreover, if the metrics are of class $C_{l o c}^{k, \alpha}$ with $k+\alpha>0$, then the diffeomorphism $\phi$ is of class $C_{l o c}^{k+1, \alpha}$.

It is important to note that the Binet-Legendre metric $g_{F}$ of a Finsler metric $F$ may be smooth even if the metric $F$ is of low regularity. A simple example is a Minkowski metric on $\mathbb{R}^{n}$ with polyhedral unit ball. This Finsler metric is not of class $C^{1}$, but its associated Binet-Legendre metric is an Euclidean metric and is therefore $C^{\infty}$. Other examples are given by the Funk metrics and, more generally, the so called Zermelo metrics, see [18, §5.2]. Additional examples are given by the class of partially smooth Finsler metrics introduced in [19, §2], see also \$5 below.

The paper is organized as follows. In $\$ 2$ we first recall some basic facts on Finsler structures and we give the definition of Binet-Legendre metric and its basic properties. We then state the optimal regularity result for Riemannian isometries due to Yu. Reshetnyak, I. Sabitov, M. Taylor, A. Lytchak and A. Yaman. In 3 we prove Theorem A and we discuss a generalization to 1-quasiconformal maps between Finsler manifolds. In 4 we recall some fine properties of Hölder continuous maps and in $\$ 5$ we use them to prove Theorem B and give some sufficient conditions for the regularity of the Binet-Legendre metric. In $\$ 6$ we give an application to Finsler manifolds admitting non trivial dilations and discuss the group of isometries of a Finsler manifold. The final section contains a brief history of the Myers-Steenrod Theorem for Riemannian manifolds and references on the subject.

We would like to thank CY Guo, S. Ivanov, A. Lytchak, D. Repovš, I. Kh. Sabitov, and E. Ščepin, for useful discussions. We are particularly grateful to A. Lytchak for attracting our interest to the paper [28].

\section{Preliminaries}

2.1. Finsler metrics. A Finsler structure on a domain $1 \mathcal{U} \subset \mathbb{R}^{n}$ is a continuous function $F: \mathcal{U} \times \mathbb{R}^{n} \rightarrow[0, \infty)$ such that for any $x \in \mathcal{U}$ and any $v, w \in \mathbb{R}^{n}$ we have

(a) $F(x, \lambda \cdot v)=\lambda \cdot F(x, v)$ for any $\lambda \geq 0$.

(b) $F(x, v+w) \leq F(x, v)+F(x, w)$.

(c) $F(x, v)=0 \Rightarrow v=0$.

Before proceeding, let us recall a few more definitions:

\footnotetext{
${ }^{1}$ Finsler structure are more generally defined as continuous maps $F$ on the tangent bundle of a $C^{1}$ manifold $M$ satisfying the conditions (a), (b) and (c) (for a domain $\mathcal{U} \subset \mathbb{R}^{n}$, we identify $T \mathcal{U}$ with $\mathcal{U} \times \mathbb{R}^{n}$ ). To define a $C^{k, \alpha}$ Finlser metric on a manifold, we need to assume that an atlas of class $C^{2} \cap C^{k+1, \alpha}$ is given on the manifold.
} 
THE MYERS-STEENROD THEOREM FOR FINSLER MANIFOLDS OF LOW REGULARITY. 3

\section{Definitions}

i) The Finsler structure $F$ on $\mathcal{U}$ is said to be reversible if $F(x,-v)=F(x, v)$ for any $(x, v) \in \mathcal{U} \times \mathbb{R}^{n}$.

ii) If the Finsler structure $F$ is independent from the point $x \in \mathcal{U}$, one says that it is a Minkowski norm.

iii) The Finsler structure is said to be of class $C^{k, \alpha}$ if the restriction of $F$ to an open neighborhood of $\mathcal{U} \times S^{n-1}=\left\{(x, v) \in \mathcal{U} \times \mathbb{R}^{n}|| v \mid=1\right\}$ is a function of class $C^{k, \alpha}$.

Remarks (i) Observe that a Minkowski norm on $\mathbb{R}^{n}$ is a norm in the usual sense if and only if it is reversible.

(ii) It is often assumed in the Finsler literature (but not in the present paper) that the Finsler structure is of class at least $C^{2}$ and for any $(x, v) \in \mathcal{U} \times\left(\mathbb{R}^{n} \backslash\{0\}\right)$ the vertical Hessian matrix

$$
\left(\frac{\partial^{2} F^{2}}{\partial v_{i} \partial v_{j}}\right)
$$

is positive definite. Such metrics are called strongly convex. Note that most results that have been proved so far on the smoothness of Finlser isometries assume the metrics to be strongly convex, see $\$ 7$

Given a Finsler structure $F$ on $\mathcal{U} \in \mathbb{R}^{n}$, one defines the $F$-length of a $C^{1}$-curve $\gamma:[a, b] \rightarrow \mathcal{U}$ as

$$
\ell_{F}(\gamma)=\int_{a}^{b} F(\gamma(t), \dot{\gamma}(t)) d t
$$

The length of any curve is invariant under sense preserving reparametrisation, it is also invariant under sense reversing reparametrisation if the Finsler structure is reversible. The distance between two points $x$ and $y$ in $\mathcal{U}$ is defined as the infimum of the length of all $C^{1}$ curves joining them

$$
d_{F}(x, y)=\inf \left\{\ell_{F}(\gamma) \mid \gamma \in C^{1}([0,1], \mathcal{U}), \gamma(0)=x, \gamma(1)=y\right\} .
$$

The distance satisfies $d_{F}(x, y)+d_{F}(y, z) \geq d_{F}(x, z)$ and $d_{F}(x, y)=0$ if and only if $x=y$. If the Finsler structure is reversible, then the distance is also symmetric: $d_{F}(x, y)=d_{F}(y, x)$.

Lemma 2.1. Let $\mathcal{U}$ be a convex domain in $\mathbb{R}^{n}$ and $F$ be a $C^{0}$ Finsler metric on $\mathcal{U}$. Suppose that there exists a constant $C>0$ such that

$$
\frac{1}{C}|v| \leq F(x, v) \leq C|v|
$$

for all $(x, v) \in \mathcal{U} \times \mathbb{R}^{n}$, where $|\cdot|$ denotes the usual Euclidean norm. Then the Finsler distance is bilipschitz equivalent to the Euclidean distance in $\mathcal{U}$, more precisely we have

$$
\frac{1}{C}|q-p| \leq d_{F}(p, q) \leq C|q-p|
$$

for any $p, q \in \mathcal{U}$.

Proof. Fix two points $p$ and $q$ in $\mathcal{U}$ and choose a $C^{1}$ path $\gamma:[0,1] \rightarrow \mathcal{U}$ joining them. We then have

$$
|q-p| \leq \int_{0}^{1}|\dot{\gamma}(t)| d t \leq C \int_{0}^{1} F(\gamma(t), \dot{\gamma}(t)) d t
$$


Taking the infimum of this inequality on all paths from $p$ to $q$ one obtains $|q-p| \leq$ $d_{F}(p, q)$. To prove the other inequality, we consider the segment $\alpha(t)=t q+(1-t) p$, this path is contained in $\mathcal{U}$ since this is a convex domain. We then have

$$
d_{F}(p, q) \leq \int_{0}^{1} F(\alpha(t), \dot{\alpha}(t)) d t \leq C \int_{0}^{1}|\dot{\alpha}(t)| d t=C \int_{0}^{1}|q-p| d t=C|q-p| .
$$

Remark. This lemma also holds for a quasi-convex domain but with a different constant in (2.1). Recall that a domain $\mathcal{U}$ in $\mathbb{R}^{n}$ is quasi-convex if there is a constant $K$ such that for any $x, y \in \mathcal{U}$ there exists a path in $\mathcal{U}$ joining $x$ to $y$ of Euclidean length at most $K|y-x|$.

2.2. The Binet-Legendre metric associated to a Finsler structure. Given a Finsler structure $F$ on $\mathcal{U}$, one defines for any point $x \in \mathcal{U}$ the associated Finsler unit tangent ball

$$
\Omega_{x}=\left\{v \in \mathbb{R}^{n} \mid F(x, v)<1\right\} .
$$

The boundary of $\Omega_{x}$ is often called the indicatrix of $F$ at $x$.

Definition The Binet-Legendre metric associated to the Finsler structure $F$ on $\mathcal{U} \subset \mathbb{R}^{n}$ is the Riemannian metric whose metric tensor $g_{i j}$ is defined at any point $x \in \mathcal{U}$ to be the inverse matrix of

$$
g^{i j}(x)=\frac{(n+2)}{\lambda^{n}\left(\Omega_{x}\right)} \int_{\Omega_{x}} v_{i} v_{j} d v_{1} \ldots d v_{n},
$$

where $\lambda^{n}\left(\Omega_{x}\right)$ is the Lebesgue measure of $\Omega_{x} \subset \mathbb{R}^{n}$.

We refer to [18 for an intrinsic (coordinate free) definition of the Binet-Legendre metric, discussions of its properties and various applications to Finsler geometry. This metric first appeared in the work of Paul Centore [5], who called it the osculating metric.

It will be useful to rewrite formula (2.2) in polar coordinates. Let us write $v=r u$ with $u \in S^{n-1}$ and $r \in \mathbb{R}_{+}$. We then have

$$
\lambda^{n}\left(\Omega_{x}\right)=\int_{\Omega_{x}} d v=\int_{S^{n-1}}\left(\int_{0}^{1 / F(x, u)} r^{n-1} d r\right) d \sigma(u)=\frac{1}{n} \int_{S^{n-1}} \frac{d \sigma(u)}{F(x, u)^{n}},
$$

where $d \sigma$ is the standard volume form on $S^{n-1}$. this proves that $x \mapsto \lambda^{n}\left(\Omega_{x}\right)$ is of class $C^{k, \alpha}$.

We likewise have

$$
\begin{aligned}
\int_{\Omega_{x}} v_{i} v_{j} d v & =\int_{S^{n-1}}\left(\int_{0}^{1 / F(x, u)}\left(r^{2} u_{i} u_{j}\right) r^{n-1} d r\right) d \sigma(u) \\
& =\int_{S^{n-1}} u_{i} u_{j}\left(\int_{0}^{1 / F(x, u)} r^{n+1} d r\right) d \sigma(u) \\
& =\frac{1}{(n+2)} \int_{S^{n-1}} \frac{u_{i} u_{j}}{F(x, u)^{n+2}} d \sigma(u) .
\end{aligned}
$$


It then follows that (2.2) can be written as

$$
g^{i j}(x)=\frac{n \int_{S^{n-1}} u_{i} u_{j} F(x, u)^{-(n+2)} d \sigma}{\int_{S^{n-1}} F(x, u)^{-n} d \sigma} .
$$

Some basic properties of the Binet-Legendre metric are stated and proved in [18]. Let us recall that if the Finsler metric $F$ on $\mathcal{U}$ is Riemannian, that is if there exists a Riemannian metric $h$ on $\mathcal{U}$ such that $F(x, v)=\sqrt{h_{x}(v, v)}$ for all $(x, v) \in \mathcal{U} \times \mathbb{R}^{n}$, then the Binet-Legendre metric of $F$ coincide with $h$.

2.3. Regularity of isometries in the Riemannian case. We will use the following result on Riemannian manifolds:

Theorem 2.2. Let $\left(\mathcal{U}_{1}, g_{1}\right)$ and $\left(\mathcal{U}_{2}, g_{2}\right)$ be two domains of $\mathbb{R}^{n}$ equipped with Riemannian metrics $g_{1}$ and $g_{2}$ of class $C^{k, \alpha}$ with $k \in \mathbb{N} \cup\{0\}, 0 \leq \alpha \leq 1$ and $k+\alpha>0$. Let $\phi:\left(\mathcal{U}_{1}, g_{1}\right) \rightarrow\left(\mathcal{U}_{2}, g_{2}\right)$ be a bijective mapping. Then, the following conditions are equivalent:

(1) $\phi$ is a distance-preserving homeomorphism,

(2) $\phi$ is bi-Lipschitz and $\phi^{*} g_{2}=g_{1}$ almost everywhere,

(3) $\phi$ is a diffeomorphism of class $C^{r+1, \alpha}$ and $\phi^{*} g_{2}=g_{1}$.

This theorem is the combined result of the work of several authors, we explain this now and give a historical overview in section 7 below.

Sketch of proof. The implication $(1) \Rightarrow(2)$ holds in fact even for $C^{0}$ metrics $g_{1}$ and $g_{2}$ as explained in the first step of the proof of [32, Theorem 2.1], and (3) $\Rightarrow$ (1) is obvious. The main issue is therefore the implication $(2) \Rightarrow(3)$.

If $k=0$ and $0<\alpha<1$, then the implication (2) $\Rightarrow(3)$ has been proved by Yu. Reshetnyack in [27, Theorem 2] (where in fact he proves a more general statement). An independant proof is given by M. Taylor in 32.

If $k+\alpha=1$, that is either $k=0$ and $\alpha=1$, or $k=1$ and $\alpha=0$, then the same implication is proved by I. Sabitov in [28].

The general case $k \geq 1$ and $0 \leq \alpha \leq 1$ follows now from the following argument of Calabi and Hartman [4, §5]. Since $k \geq 1$, the map $\phi$ is $C^{2}$ and satisfy $\phi^{*} g_{2}=g_{1}$ from the previous case. Recall now that in any local coordinates systems, the Christoffel symbols $\tilde{\Gamma}_{\nu \lambda}^{m}$ and $\tilde{\Gamma}_{\nu \lambda}^{m}$ of the metrics $g_{1}$ and $g_{2}$ are related by the formula

$$
\frac{\partial^{2} \phi^{m}}{\partial x^{i} \partial x^{j}}=\Gamma_{i j}^{\mu} \frac{\partial \phi^{m}}{\partial x^{\mu}}-\tilde{\Gamma}_{\nu \lambda}^{m} \frac{\partial \phi^{\nu}}{\partial x^{i}} \frac{\partial \phi^{\lambda}}{\partial x^{j}} .
$$

Since the Christoffel symbols are given by algebraic expressions involving the first derivatives of the metric tensors $g_{1}$ and $g_{2}$, the implication $(2) \Rightarrow(3)$ follows from (2.4) by induction on $k$ for any given $0 \leq \alpha \leq 1$.

Remarks 1.) This Theorem is an optimal regularity result for isometries of $C^{k, \alpha}$ Riemannian metrics, which of course implies that our Theorem A, and hence Corollary C, are also optimal. Indeed, E. Calabi and P. Hartman have built an example of a non differentiable isometry between two $C^{0}$ Riemannian metrics, see [4. §6]. 
2.) The result is also optimal in the sense that one cannot expect an isometry between $C^{k, \alpha}$ metrics to be more than $C^{k+1, \alpha}$-regular. A simple (Riemannian) example can be build as follows: choose a diffeomorphism $f: \mathbb{R} \rightarrow \mathbb{R}$ which is of class $C^{k+1, \alpha}$ but does not belong to $C^{k+1, \beta}$ for any $\beta>\alpha$. Consider the following Riemannian metrics on the plane:

$$
g_{1}=f^{\prime}\left(x_{1}\right)^{2} d x_{1}^{2}+d x_{2}^{2} \quad \text { and } \quad g_{2}=d x_{1}^{2}+d x_{2}^{2} .
$$

Then the map $\phi:\left(x_{1}, x_{2}\right) \mapsto\left(f\left(x_{1}\right), x_{2}\right)$ is an isometry from $\left(\mathbb{R}^{2}, g_{1}\right)$ to $\left(\mathbb{R}^{2}, g_{2}\right)$. By construction, the map $\phi$ is of class $C^{k+1, \alpha}$ but not of class $C^{k+1, \beta}$ for any $\beta>\alpha$.

3.) Note that the implication $(2) \Rightarrow(3)$ fails if we only assume the map $\phi$ to be differentiable almost everywhere. Consider for instance 2 the map from the unit square $(0,1)^{2}$ to $\mathbb{R}^{2}$ defined by $\phi(x, y)=(x, y+\nu(x))$, where $\nu:(0,1) \rightarrow(0,1)$ is the Cantor-Lebesgue function. Then $\phi$ is a homeomorphism onto its image such that $\phi^{*} g_{0}=g_{0}$ a.e. where $g_{0}=d x^{2}+d y^{2}$ is the standard Euclidean metric, yet $\phi \notin C^{1}$. However, Reshetnyak's theorem tells us that assuming $\phi \in W^{1, n}$ is sufficient for the implication $(2) \Rightarrow(3)$.

\section{Proof of Theorem A}

We first observe that our hypothesis, together with Lemma 2.1. imply that the map $\phi$ is bilipschitz. It then follows from Rademacher's theorem that $\phi$ is (Frechet) differentiable almost everywhere [9, §3.1.2].

We claim that the following condition holds at any point $x \in \mathcal{U}_{1}$ where $\phi$ is differentiable:

$$
F_{1}(x, v)=F_{2}\left(\phi(x), d \phi_{x}(v)\right), \quad\left(\forall v \in \mathbb{R}^{n}\right) .
$$

To prove the claim, we may suppose that $\mathcal{U}_{1}$ and $\mathcal{U}_{2}$ contain the origin and that $\phi(0)=0$. Suppose also that $\phi$ is differentiable at $x=0$ and let us introduce the following notations (for $t>0$ and $i=1,2$ )

$$
\mathcal{U}_{i, t}=\frac{1}{t} \mathcal{U}_{i}, \quad d_{i, t}(x, y)=\frac{1}{t} d_{i}(t x, t y), \quad F_{i, t}(x, v)=F_{i}(t x, v)=\frac{1}{t} F_{i}(t x, t v) .
$$

Let us also define the map $\phi_{t}: \mathcal{U}_{1, t} \rightarrow \mathcal{U}_{2, t}$ by $\phi_{t}(x)=\frac{1}{t} \phi(t x)$. From the definitions, we see that $d_{i, t}(x, y)$ is the distance on $\mathcal{U}_{i, t}$ associated to the Finsler metric $F_{i, t}(x, v)$. It is also easy to check that $\phi_{t}$ is a distance preserving bijection from $\left(\mathcal{U}_{1, t}, d_{1, t}\right)$ to $\left(\mathcal{U}_{2, t}, d_{2, t}\right)$.

Since we assumed that $\phi$ is differentiable at 0 , the limit $\phi_{0}=\lim _{t \rightarrow 0} \phi_{t}$ exists uniformly, and it is a map from $\mathbb{R}^{n}$ to $\mathbb{R}^{n}$. In fact we have $\phi_{0}=d \phi_{0}$ (the differential of $\phi$ at 0 ), in particular it is a linear map.

By continuity $\phi_{0}$ is an isometry for the limit distances $d_{1,0}=\lim _{t \rightarrow 0} d_{1, t}$ and $d_{2,0}=\lim _{t \rightarrow 0} d_{2, t}$. These distances are associated to the Finsler metrics $F_{i, 0}(x, v)=$ $\lim _{t \rightarrow 0} F_{i, t}(x, v)=F_{i}(0, v)$. Note that in fact $F_{1,0}$ and $F_{2,0}$ are Minkowski metric on $\mathbb{R}^{n}$. This implies that $d \phi_{0}=\phi_{0}$ is an isometry between two Minkowski spaces $\left(\mathbb{R}^{n}, F_{1,0}\right)$ and $\left(\mathbb{R}^{n}, F_{2,0}\right)$. The claim is proved.

Let us denote by $g_{i}$ the Binet-Legendre metric of $F_{i}$. By hypothesis, this is a $C^{k, \alpha}$ Riemannian metric on $\mathcal{U}_{i}$. It follows now from the claim and standard properties of the Binet-Legendre metric that

$$
\left.g_{2}\right|_{\phi(x)}(d \phi(v), d \phi(w))=\left.g_{1}\right|_{x}(v, w),
$$

\footnotetext{
${ }^{2}$ We owe this example to Changyu Guo.
} 
for a.e. $x \in \mathcal{U}_{1}$ and all $v, w \in \mathbb{R}^{n}$.

We thus have proved that $g_{i}$ is a $C^{k, \alpha}$ Riemannian metric on $\mathcal{U}_{i}$ for $i=1,2$ and $\phi: \mathcal{U}_{1} \rightarrow \mathcal{U}_{2}$ is a bilipschitz map such that $\phi^{*} g_{2}=g_{1}$ almost everywhere. By Theorem 2.2 we conclude that $\phi \in C^{k+1, \alpha}\left(\mathcal{U}_{1}, \mathcal{U}_{2}\right)$. Theorem A is proved.

3.1. Extension of Theorem A to the case of 1-quasiconformal maps. In this section we briefly explain how to extend our results to the case of quasiconformal maps. Recall that a homeomorphism $\phi: X \rightarrow Y$ between two metric measure spaces $(X, d)$ and $\left(Y, d^{\prime}\right)$ is said to be quasiconformal if the linear distorsion, defined as

$$
H(x, \phi)=\limsup _{r \rightarrow 0} \frac{\max \left\{d^{\prime}(\phi(x), \phi(z)) \mid z \in B(x, r)\right\}}{\min \left\{d^{\prime}(\phi(x), \phi(z)) \mid z \in B(x, r)\right\}}
$$

is uniformly bounded in $X$. The map is said to be 1-quasiconformal if $H(x, \phi)=1$ a.e. A regularity theorem for Riemannian 1-quasiconformal has been proved by Reshetnyak in 27. See also [1] and 14. Using this result we can state the following

Theorem D. Let $\phi:\left(M_{1}, F_{1}\right) \rightarrow\left(M_{2}, F_{2}\right)$ be a 1-quasiconformal map between two Finsler manifolds. Assume that the Binet-Legendre metrics $g_{1}$ and $g_{2}$ of $F_{1}$ and $F_{2}$ belong to $C_{\text {loc }}^{k, \alpha}$, where $k \in \mathbb{N} \cup\{0\}, 0 \leq \alpha<1$ and $k+\alpha>0$. Then $\phi \in C_{l o c}^{k+1, \alpha}\left(M_{1}, M_{2}\right)$ and $\phi^{*}\left(F_{2}\right)=F_{1}$. In particular, the map $\phi$ is conformal.

Sketch of proof. The notion of quasiconformality is invariant under a bilipschitz change of the metrics, therefore the restriction of the quasi-conformal map $\phi$ : $M_{1} \rightarrow M_{2}$ to any coordinate neighborhood is also quasiconformal with respect to the Euclidean metric. Using the standard theory of quasiconformal maps in $\mathbb{R}^{n}$, one then deduces that $\phi$ is differentiable almost everywhere and that its differential is a.e. invertible (see e.g. [12, Chapter 6], the differentiability a.e. follows from Rademacher-Stepanov's theorem and the fact that the differential is a.e. invertible follows form the change of variable formula in integrals). Now using a blow-up argument as in the beginning of the proof of Theorem A, we obtain

$$
\max _{F_{1}(x, u)=1} F_{2}\left(\phi(x), d \phi_{x}(u)\right)=H(x, \phi) \cdot \min _{F_{1}(x, u)=1} F_{2}\left(\phi(x), d \phi_{x}(u)\right)
$$

at any point $x \in M_{1}$ where $\phi$ is differentiable with invertible differential. In particular, if $\phi: M_{1} \rightarrow M_{2}$ is 1-quasiconformal, then the following equality holds for almost all $x \in M_{1}$ and all $v \in T_{x} M$

$$
F_{2}\left(\phi(x), d \phi_{x}(v)\right)=\mu(x) F(x, v),
$$

where

$$
\mu(x)=\max _{F_{1}(x, u)=1} F_{2}\left(\phi(x), d \phi_{x}(u)\right)=\min _{F_{1}(x, u)=1} F_{2}\left(\phi(x), d \phi_{x}(u)\right) .
$$

The above identity can also be written as $\phi^{*} F_{2}=\mu F_{1}$ a.e. This implies that $\phi^{*} g_{2}=\mu g_{1}$ a.e., that is $\phi:\left(M_{1}, g_{1}\right) \rightarrow\left(M_{2}, g_{2}\right)$ is Riemannian 1-quasiconformal. We conclude from [27, Theorem 2] together with [14, Theorem 4.5] or [11, 23] that $\phi \in C_{l o c}^{k+1, \alpha}$. 


\section{ON HÖLDER AND LIPSCHITZ MAPS}

In this section we collect some facts on Hölder continuous and Lipschitz mappings that we will need later. Let $\mathcal{U}$ be a domain in $\mathbb{R}^{n}$ and $0<\alpha<1$. A map $f: \mathcal{U} \rightarrow \mathbb{R}^{m}$ is said to be Hölder continuous of class $C^{0, \alpha}$ if

$$
[f]_{C^{0, \alpha}(\mathcal{U})}=\sup \left\{\frac{|f(y)-f(x)|}{|y-x|^{\alpha}} \mid x, y \in \mathcal{U}, x \neq y\right\}<\infty .
$$

Here $|y-x|$ denotes the standard Euclidean distance between $x$ and $y$. The map $f$ is said to be Lipschitz if the same condition holds with $\alpha=1$.

A map $f: \mathcal{U} \rightarrow \mathbb{R}^{m}$ is then said to be of class $C^{k, \alpha}$, where $0<\alpha<1$ and $k \in \mathbb{N}$ if $f \in C^{k}\left(\mathcal{U}, \mathbb{R}^{m}\right)$ and all partial derivatives of order $k$ are $\alpha$-Hölder continuous. This is a Banach space for the norm

$$
\|f\|_{C^{k, \alpha}(\mathcal{U})}=\|f\|_{C^{k}(\mathcal{U})}+\max _{\beta}\left[D^{\beta} f\right]_{C^{0, \alpha}(\mathcal{U})},
$$

where $\beta \in \mathbb{N}^{n}$ runs through all multi-indices of order $k$. Concerning these spaces we will need the following results:

Proposition 4.1. Let $\mathcal{U} \subset \mathbb{R}^{n}$ be a bounded domain with Lipschitz boundary and fix $k \in \mathbb{N} \cup\{0\}$ and $0 \leq \alpha \leq 1$.

(A) If $f_{1}, f_{2} \in C^{k, \alpha}(\mathcal{U}, \mathbb{R})$, then we also have $f_{1} f_{2} \in C^{k, \alpha}(\mathcal{U}, \mathbb{R})$ and

$$
\left\|f_{1} f_{2}\right\|_{C^{k, \alpha}(\mathcal{U})} \leq C\left(\left\|f_{1}\right\|_{C^{0}(\mathcal{U})}\left\|f_{2}\right\|_{C^{k, \alpha}(\mathcal{U})}+\left\|f_{2}\right\|_{C^{0}(\mathcal{U})}\left\|f_{1}\right\|_{C^{k, \alpha}(\mathcal{U})}\right)
$$

for some constant $C=C(\mathcal{U}, k)$.

(B) If $f \in C^{k, \alpha}(\mathcal{U}, \mathbb{R})$ and $f \geq a>0$ in $\mathcal{U}$, then $1 / f \in C^{k, \alpha}(\mathcal{U}, \mathbb{R})$ and

$$
\left\|\frac{1}{f}\right\|_{C^{k, \alpha}(\mathcal{U})} \leq C \cdot a^{-(k+2)}\|f\|_{C^{0}(\mathcal{U})}\|f\|_{C^{k, \alpha}(\mathcal{U})}
$$

for some constant $C=C(\mathcal{U}, k)$

The proof is given in [6, Theorems 16.28 and 16.29].

Proposition 4.2. Let $\mathcal{U} \subset \mathbb{R}^{n}$ be a an open set and $(S, \mu)$ be an arbitrary measure space. Let $h: \mathcal{U} \times S \rightarrow \mathbb{R}$ be a measurable function such that for a.e. $s \in S$ we have

$$
\left\|h_{s}\right\|_{C^{k, \alpha}(\mathcal{U})} \leq m(s),
$$

where $h_{s}(x)=h(x, s)$ and $m \in L^{1}(S, d \mu)$, then the function $H: \mathcal{U} \rightarrow \mathbb{R}$ defined by

$$
H(x)=\int_{S} h(x, s) d s
$$

belongs to $C^{k, \alpha}(\mathcal{U})$ and satisfies $\|H\|_{C^{k, \alpha}(\mathcal{U})} \leq\|m\|_{L^{1}(S)}$.

Proof. Let us first consider the case $k=0$, we have for any pair of distinct points $x$ and $y$ in $\mathcal{U}$

$$
\frac{|H(y)-H(x)|}{|y-x|^{\alpha}} \leq \int_{S} \frac{\mid h((y, s)-h(x, s) \mid}{|y-x|^{\alpha}} d \mu(s) .
$$

On the other hand, using Lebesgue's dominated convergence theorem, we easily prove that

$$
\|H\|_{C^{k}(\mathcal{U})} \leq \int_{S}\left\|h_{s}\right\|_{C^{k}(\mathcal{U})} d \mu(s) .
$$


From the two inequalities above, we conclude that

$$
\|H\|_{C^{k, \alpha}(\mathcal{U})} \leq \int_{S}\left\|h_{s}\right\|_{C^{k, \alpha}(\mathcal{U})} d \mu(s) \leq\|m\|_{L^{1}(S)} .
$$

We also have the following result on composition (see Theorem 16.31 in [6]).

Theorem 4.3. Let $\mathcal{U} \subset \mathbb{R}^{n}$ and $\mathcal{V} \subset \mathbb{R}^{m}$ be bounded open sets with Lipschitz boundaries, and let $h \in C^{k, \alpha}\left(\mathcal{V}, \mathbb{R}^{p}\right)$ and $f \in C^{k, \alpha}(\mathcal{U}, \mathcal{V})$. Assume also that $f$ is Lipschitz continuous, then $h \circ f \in C^{k, \alpha}\left(\mathcal{U}, \mathbb{R}^{p}\right)$.

\section{Regularity of the Binet-Legendre metric}

Theorem A would be an empty shell without concrete criteria implying the $C^{k, \alpha}$ regularity of the Binet-Legendre metric. The goal of this section is precisely to provide such criteria. We first proof Theorem B which is a result for $C^{k, \alpha}$ Finsler metric and then we discuss a generalized regularity condition, called $(k, \alpha)$-partial smoothness, for Finsler metric and we prove a generalization of Theorem B for this class of metrics.

Proof of Theorem B. We can assume without loss of generality that $\mathcal{U} \subset \mathbb{R}^{n}$ is a bounded convex domain and $F: \mathcal{U} \times \mathbb{R}^{n} \rightarrow \mathbb{R}$ is a continuous Finsler structure such that

$$
\frac{1}{C_{0}}|v| \leq F(x, v) \leq C_{0}|v|
$$

for some constant $C_{0}>0$ and all $(x, v) \in \mathcal{U} \times \mathbb{R}^{n}$. Using Formula (2.3) and Proposition 4.1, we conclude from that $x \mapsto g^{i j}(x)$ is a function of class $C^{k, \alpha}$. The condition (5.1) on $F$ implies also that $\operatorname{det}\left(g^{i j}\right)$ is bounded below away from zero (see e.g. [18, Proposition 12.1]). Using Proposition 4.1 again, we then conclude that the inverse matrix $g_{i j}(x)$ is also a $C^{k, \alpha}$ function of $x$ (see also Corollary 16.30 in [6]).

We will generalize Theorem B to more general Finsler metrics. To this aim we need the following

Definition. Let $k \in \mathbb{N} \cup\{0\}$ and $\alpha \in[0,1]$. Let $F$ be a continuous Finsler metric on the domain $\mathcal{U} \subset \mathbb{R}^{n}$. We say that the metric $F$ is $(k, \alpha)$-partially smooth, if there exists a map $A: \mathcal{U} \times \mathbb{R}^{n} \rightarrow \mathbb{R}^{n}$ such that

i.) $A(x, \lambda v)=\lambda A(x, v)$ for all $(x, v) \in \mathcal{U} \times \mathbb{R}^{n}$ and all $\lambda \geq 0$;

ii.) For any $x \in \mathcal{U}$, the map $A_{x}: \mathbb{R}^{n} \rightarrow \mathbb{R}^{n}$ defined by $v \mapsto A(x, v)$ is bilipschitz;

iii.) There exists a constant $C>0$, such that

$$
\left\|h_{u}\right\|_{C^{k, \alpha}(\mathcal{U})}+\left\|A_{u}\right\|_{C^{k, \alpha}(\mathcal{U})}+\left\|J_{u}\right\|_{C^{k, \alpha}(\mathcal{U})} \leq C
$$

for all $u \in S^{n-1}$, where $3 A_{u}(x)=A(x, u), J_{u}(x)=J(x, u)=\left|\operatorname{det}\left(D A_{x}\right)(u)\right|$ is the Jacobian of the map $A_{x}$ and $h_{u}(x)=F(x, A(x, u))$.

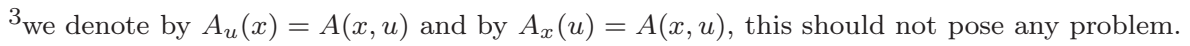


Note that this notion of partial smoothness is slightly different from (but strongly related to) the notion of $C^{k}$-partial-smoothness introduced in [18, Definition 2.1.]. Observe also that if we assume the maps $A_{x}$ to be the identity, that is $A(x, v)=v$, then this condition reduces to the standard $C^{k, \alpha}$ regularity. In the general case, the role of the map $A(x, v)$ is to allow a Finsler metric to be regular in $x$ after twisting the parameter $v$, see [18, §2] for some explanations and explicit examples.

Theorem B'. Let $\mathcal{U}$ be a bounded convex domain in $\mathbb{R}^{n}$ and let $F: \mathcal{U} \times \mathbb{R}^{n} \rightarrow \mathbb{R}$ be a $(k, \alpha)$ partially smooth Finsler metric in $\mathcal{U}$ satisfying the inequalities (5.1) for some constant $C_{0}$. Then the Binet-Legendre metric of $F$ is of class $C^{k, \alpha}$.

Note that this result generalizes Theorem B.

Proof. The argument will be based on Proposition 4.1 together with a twisted version of (2.3). Let us set for any $x \in \mathcal{U}$

$$
\Omega_{x}^{\prime}=A_{x}^{-1}\left(\Omega_{x}\right)=\left\{v^{\prime} \in \mathbb{R}^{n} \mid F\left(x, A_{x}\left(v^{\prime}\right)\right)<1\right\} .
$$

We then have with $J\left(x, v^{\prime}\right)=\left|\operatorname{det}(D A)_{x}\left(v^{\prime}\right)\right|$

$$
\begin{aligned}
\lambda\left(\Omega_{x}\right) & =\int_{\Omega_{x}^{\prime}} J\left(x, v^{\prime}\right) d v^{\prime} \\
& =\int_{S^{n-1}}\left(\int_{0}^{1 / F(x, A(u))} J(x, r u) r^{n-1} d r\right) d u \\
& =\frac{1}{n} \int_{S^{n-1}} \frac{J(x, u)}{F\left(x, A_{x}(u)\right)^{n-1}} d \sigma(u),
\end{aligned}
$$

where we have used polar coordinates $v^{\prime}=r u$, with $u \in S^{n-1}$. We have also used the fact that $A_{x}$ is homogenous of degree 1 and therefore $J(x, r u)=J(x, u)$.

The above identity controls the denominator in the definition of the Binet-Legendre metric. To obtain a similar formula for the numerator, we denote by

$$
a_{i}\left(x, v^{\prime}\right)=\left(A\left(x, v^{\prime}\right)\right)_{i}
$$

the $i^{\text {th. }}$ coordinate of $A\left(x, v^{\prime}\right)$. We then have

$$
\begin{aligned}
\int_{\Omega_{x}} v_{i} v_{j} d v & =\int_{\Omega_{x}^{\prime}} a_{i}\left(x, v^{\prime}\right) a_{j}\left(x, v^{\prime}\right) J\left(x, v^{\prime}\right) d v^{\prime} \\
& =\int_{S^{n-1}}\left(\int_{0}^{1 / F(x, A(u))} a_{i}(x, u) a_{j}(x, u) J(x, r u) r^{n+1} d r\right) d u \\
& =\frac{1}{n+2} \int_{S^{n-1}} \frac{a_{i}(x, u) a_{j}(x, u) J(x, u)}{F\left(x, A_{x}(u)\right)^{n-1}} d \sigma(u),
\end{aligned}
$$

where we have again used $v^{\prime}=r u$ and the homogeneity $a_{i}(x, r u)=r a_{i}(x, u)$. Using Propositions 4.1 and 4.2 together with the inequalities (5.1) and (5.2), we conclude as in the proof of Theorem B.

We then clearly have the following

Corollary C'. A distance preserving bijective map between two $(k, \alpha)$-partially smooth Finsler manifolds with $k+\alpha>0$ is a $C_{l o c}^{k+1, \alpha}$-diffeomorphism. 


\section{Some Applichtions of our RESUlts}

6.1. Finsler manifolds with non trivial dilation. A direct consequence of Corollary $\mathrm{C}$ is the following

Corollary 6.1. Let $(M, F)$ be a $C^{0, \alpha}$ Finsler manifold with $\alpha>0$. Assume that $F$ is forward complete and that there exists a map $\phi: M \rightarrow M$ such that $d_{F}(\phi(x), \phi(y))=a d_{F}(x, y)$ for any points $x, y \in M$ and some constant $a \neq 1$. Then $(M, F)$ is isometric to a $\mathbb{R}^{n}$ with a Minkowski metric and the isometry from $M$ to $\mathbb{R}^{n}$ is a diffeomorphism.

Proof. The map $\phi$ is a distance preserving map from $(M, a F)$ to $(M, F)$. Since $F$ is Hölder continuous, one concludes from Corollary $\mathrm{C}$ that $\phi$ is of class $C^{1, \alpha}$ and $\phi^{*} F=a F$. The results follows then from Theorem 6.1 in [19].

6.2. On the group of isometries. Let $(M, F)$ be a $C^{0}$ connected Finsler manifold and $\operatorname{Isom}\left(M, d_{F}\right)$ the group of its distance preserving bijective maps $\phi: M \rightarrow M$. We then have

Proposition 6.2. The group $\operatorname{Isom}\left(M, d_{F}\right)$ is locally compact for the compact open topology.

Proof. If the map $\phi: M \rightarrow M$ preserves the Finsler distance $d_{F}$, then it also preserves its symmetrization $\rho(x, y)=\frac{1}{2}\left(d_{F}(x, y)+d_{F}(y, x)\right)$. By a theorem of Dantzig and van der Waerden, we know that $\operatorname{Isom}(M, \rho)$ is locally compact for the compact open topology, see e.g. [13, Theorem 4.7 in Chapter 1]. The result follows since $\operatorname{Isom}\left(M, d_{F}\right)$ is clearly a closed subgroup of $\operatorname{Isom}(M, \rho)$.

We now conclude that the isometry group of a mildly regular Finsler manifold is a Lie group.

Theorem 6.3. Let $(M, F)$ be a smooth connected $C^{0}$ Finsler manifold. Assume that its Binet-Legendre metric is of class $C^{0, \alpha}$ with $\alpha>0$. Then the group of isometries of $\left(M, d_{F}\right)$ is a Lie group for the compact open topology, and its action on $M$ is by diffeomorphisms.

Proof. We just proved that $\operatorname{Isom}\left(M, d_{F}\right)$ is locally compact for the compact open topology. Since every element $\phi \in \operatorname{Isom}\left(M, d_{F}\right)$ is of class $C^{1}$, we conclude from a classical theorem of Montgomery and his collaborators, see [3] and [21, Chapter 5], that $\operatorname{Isom}\left(M, d_{F}\right)$ is a Lie group. By Corollary $\mathrm{C}$, it acts by diffeomorphisms.

\section{Remarks.}

a.) Actually, the isometry group of a Finsler manifold is a Lie group even if the Finsler metric is only of class $C^{0}$ (and without assuming any regularity of the Binet-Legendre metric). This follows from the proof of the Hilbert-Smith conjecture for Lipschitz homeomorphisms given in [26]. The general HilbertSmith conjecture states that a locally compact topological group $G$ that acts faithfully on a connected manifold $M$ is a Lie group, Recent references are [16, 20, 21]. The conjecture has been recently proved in dimension 3 in [24]. 
b.) Another particular case of the Hilbert-Smith conjecture is due to G. Martin [17]. It states that a locally compact group acting effectively and quasiconformally on a Riemannian manifold is a Lie group. Using the Binet-Legendre metric, this results immediately extends to the case of Finsler manifolds.

c.) If we assume the Finsler metric $F$ to be $C^{2}$, then the Binet-Legendre metric is also $C^{2}$ and Theorem 6.3 follows from [22, Theorem 10].

d.) In the case when the Finsler metric $F$ is $C^{2}$ and strongly convex, Theorem 6.3 was proved in [7] by S. Deng and Z. Hou, see also [8, §3.2].

\section{A BRIEF History of Myers-Steenrod's Theorem}

The history behind the results discussed in the present paper is somewhat intricate. The original 1939 paper of Myers and Steenrod clearly sets the problem. This paper claims that (I) every distance preserving homeomorphism of a $C^{1}$ Riemannian manifold $(M, g)$ is a $C^{1}$ diffeomorphism and that (II) any closed group of isometries of a Riemannian manifold of class $C^{2}$ is a Lie group. Both theorems are often referred to as the Myers-Steenrod Theorem.

The key idea of Myers and Steenrod to prove the first result is to represent tangent vectors in $M$ as velocity vectors of geodesics and argue that since geodesics are a metric notion, distance preserving maps send geodesics to geodesics and therefore act continuously on the unit tangent bundle of $M$, from which it follows that they are of class $C^{1}$. The argument is correct and is written with some details in a more modern language in [10. Chap.1, Theorem 11.1], but it requires the Riemannian metric to be at least of class $C^{2}$. See also [25, Theorem 9.1] for an alternative proof, also for $C^{2}$ metrics, based on systems of coordinates defined by distance functions. A readable proof of statement (II) is given in [13, Chap. 6, Theorem 3.4].

In 1970, Calabi and Hartman pointed at the necessity in Myers-Steenrod's argument to prove the existence of tangent directions for the geodesics of Riemannian metrics of low regularity and they prove a version of (I) for Hölder continuous metrics.

In 1978 however, Yu. Reshetnyak in 27, found a mistake in a regularity result for geodesics used by Calabi and Hartman in 44 (in the proof of Theorem 3.1 of that paper to be precise). This mistake was independently discovered by A. Lytchak and A. Yaman in [15], who also constructed a counterexample to a statement Calabi and Hartman used in their proof. In fact the result that can be proved by methods of Calabi and Hartman is that a distance preserving homeomorphism of a $C^{0, \alpha}$ Riemannian manifold $(0<\alpha<1)$ is a $C^{1, \frac{\alpha}{2}}$-diffeomorphism, see [28, 29] and [15] for more details.

This result was improved in Reshetnyak 27] and S. Z. Shefel' 31, who approached the problem via the theory of quasiconformal maps. In particular, Theorem 2.2 follows from [27, Theorem 2] for $k=0,0<\alpha<1$, and from [31] for $k \geq 1,0<$ $\alpha<1$. The remaining cases, when the metrics have regularity $C^{0,1}$ or $C^{1,0}$ was proved in 28 .

Another approach to this problem is due to M. Taylor who proved Theorem 2.2 for all $k \geq 0$ and $0 \leq \alpha<1$. His proof, which is elegant and self-contained, is based on harmonic coordinates and the regularity theory for elliptic PDEs (still assuming $k+\alpha>0$ ). We refer to the book 29] for more information and further developments. 
The generalization of Myers-Steenrod theorems to Finsler manifolds appears as a natural question, and, for $C^{\infty}$ smooth strongly convex metrics on manifolds of dimension at least 3, it follows from the result of F. Brickell in [2]. The result in all dimensions has then been proved in 2002 by S. Deng \& Z. Hou [7]. See also the paper B. Aradi \& D. Kertész [1] for a different point of view. All these works assume the Finsler structure $F$ to be strongly convex, which we do not do in the present paper.

On the other hand, the paper [15] by Lytchak \& Yaman also considers the case of Hölder continuous Finsler manifolds, however they need a special convexity condition on the Finsler unit ball that they call uniform convexity of type $p$, see [15], definition 2.3]. They also prove that a distance preserving homeomorphism of a $C^{\alpha}$ Finsler manifold $(0<\alpha<1)$ of type $p$ is a $C^{1, \beta}$ diffeomorphism with $\beta=\frac{\alpha}{p}$. See the remark following Theorem 1.3 in [15]. Our main Theorems extend all those previous cases and are optimal.

\section{REFERENCES}

[1] B. Aradi, and D. Kertész, Isometries, submetries and distance coordinates on Finsler manifolds. Acta Math. Hungar. 143 (2014), no. 2, 337-350.

[2] F. Brickell, On the differentiability of affine and projective transformations. Proc. Amer. Math. Soc. 16 (1965) 567-574.

[3] S. Bochner, Salomon and D. Montgomery, Locally compact groups of differentiable transformations. Ann. of Math. (2) 47, (1946). 639-653.

[4] E. Calabi and P. Hartman, On the smoothness of isometries. Duke Math. J. 371970 741-750.

[5] P. Centore, Volume forms in Finsler spaces. Houston J. Math. 25(1999), no. 4, 625-640.

[6] G. Csató, B. Dacorogna and O. Kneuss, The pullback equation for differential forms. Progress in Nonlinear Differential Equations and their Applications, 83. Birkhäuser/Springer, New York, 2012.

[7] S. Deng and Z. Hou, The group of isometries of a Finsler space. Pacific J. Math. 207 (2002), no. 1, 149-155.

[8] S. Deng, Homogeneous Finsler spaces. Springer Monographs in Mathematics. Springer, New York, 2012

[9] L. Evans and R. Gariepy, Measure theory and fine properties of functions. Studies in Advanced Mathematics. CRC, 1992.

[10] S. Helgason, Differential geometry, Lie groups, and symmetric spaces. Academic Press, Inc., New York-London, 1978.

[11] T. Iwaniec, Regularity theorems for solutions of partial differential equations for quasiconformal mappings in several dimensions. Dissertationes Math. 198 (1982).

[12] T. Iwaniec and G. Martin, Geometric function theory and non-linear analysis, Oxford University Press, 2001.

[13] S. Kobayashi and K. Nomizu, Foundations of differential geometry. Vol I. Interscience Publishers, John Wiley \& Sons, New York-London 1963.

[14] T. Liimatainen and M. Salo, n-harmonic coordinates and the regularity of conformal mappings. Math. Res. Lett. 21 (2014), no. 2, 341-361.

[15] A. Lytchak, and A. Yaman, On Hölder continuous Riemannian and Finsler metrics. Trans. Amer. Math. Soc. 358 (2006), no. 7, 2917-2926.

[16] I. Maleshich, The Hilbert-Smith conjecture for Hölder actions. Uspekhi Mat. Nauk 52 (1997), no. 2 (314), 173-174; translation in Russian Math. Surveys 52 (1997), no. 2, 407-408

[17] G. Martin, The Hilbert-Smith conjecture for quasiconformal actions. Electron. Res. Announc. Amer. Math. Soc. 5 (1999), 66-70 (electronic).

[18] V. S. Matveev and Marc Troyanov, The Binet-Legendre metric in Finsler geometry. Geom. Topol. 16(2012), no. 4, 2135-2170.

[19] V. S. Matveev and Marc Troyanov, Completeness and incompleteness of the Binet-Legendre metric. Eur. J. Math. 1 (2015), no. 3, 483-502. 
[20] George Michael, A. A. On locally Lipschitz locally compact transformation groups of manifolds. Arch. Math. (Brno) 43 (2007), no. 3, 159-162.

[21] D. Montgomery and L. Zippin, Topological transformation groups. Interscience Publishers, New York-London, 1955.

[22] S. B. Myers and N. E. Steenrod, The Group of Isometries of a Riemannian Manifold. Annals of Mathematics Vol. 40, No. 2 (1939), pp. 400-416.

[23] I. Nikolaev and S. Shefel', Differential properties of mappings that are conformal at a point (English. Russian original) Sib. Math. J. 27, 106-114 (1986); translation from Sib. Mat. Zh. 27, No. 1, 132-142 (1986).

[24] J. Pardon, The Hilbert-Smith conjecture for three-manifolds. J. Amer. Math. Soc. 26 (2013), no. 3, 879-899.

[25] P. Petersen, Riemannian geometry, second ed., Graduate Texts in Mathematics, vol. 171, Springer, NewYork, 2006.

[26] D. Repovš and E. Ščepin, A proof of the Hilbert-Smith conjecture for actions by Lipschitz maps. Math. Ann. 308 (1997), no. 2, 361-364.

[27] Ju. G. Reshetnyak, Differential properties of quasiconformal mappings and conformal mappings of Riemannian spaces. (Russian) Sibirsk. Mat. Zh. 19 (1978), no. 5, 1166-1183, 1216. Transleted in Sib. Math. J. 19, 822-834 (1979).

[28] I. Kh. Sabitov, On the smoothness of isometries. (Russian) Sibirsk. Mat. Zh. 34 (1993), no. 4, 169-176, iv, x; translation in Siberian Math. J. 34 (1993), no. 4, 741-748.

[29] I. Kh. Sabitov, Isometric immersions and embeddings of locally Euclidean metrics. Reviews in Mathematics and Mathematical Physics, 13, part 1. Cambridge Scientific Publishers, Cambridge, 2008.

[30] E. Ščepin, Hausdorff dimension and the dynamics of diffeomorphisms. (Russian) Mat. Zametki 65 (1999), no. 3, 457-463; translation in Math. Notes 65 (1999), no. 3-4, 381-385.

[31] S. Z. Shefel', Smoothness of a conformal map of Riemannian spaces. (Russian) Sibirsk. Matemat. Zh. 23 (1982), no. 1, pp. 153-159; translation in Siberian Math. J. 23 (1982), no $1,119-124$

[32] M. Taylor, Existence and regularity of isometries. Trans. Amer. Math. Soc. 358 (2006), no. 6, 2415-2423.

Institut für Mathematik, Friedrich-Schiller Universität Jena, 07737 Jena, Germany

E-mail address: vladimir.matveev@uni-jena.de

Section de Mathématiques, École Polytechnique Féderale de Lausanne, station 8, 1015 Lausanne - Switzerland

E-mail address: marc.troyanov@epfl.ch 